\title{
Corela
}

Cognition, représentation, langage

HS-4 | 2006

Le parcours

\section{Relation entre deux termes et stabilisation : le cas de want.}

\section{Olivier Polge}

\section{(2) OpenEdition}

Journals

\section{Édition électronique}

URL : http://journals.openedition.org/corela/1410

DOI : 10.4000/corela.1410

ISSN : 1638-573X

\section{Éditeur}

Cercle linguistique du Centre et de l'Ouest - CerLICO

\section{Référence électronique}

Olivier Polge, «Relation entre deux termes et stabilisation : le cas de want. », Corela [En ligne], HS-4 | 2006, mis en ligne le 08 juin 2006, consulté le 07 mai 2019. URL : http:// journals.openedition.org/corela/1410; DOI : 10.4000/corela.1410

Ce document a été généré automatiquement le 7 mai 2019.

\section{(c) (i) (2)(2)}

Corela - cognition, représentation, langage est mis à disposition selon les termes de la licence Creative Commons Attribution - Pas d'Utilisation Commerciale - Partage dans les Mêmes Conditions 4.0 International. 


\title{
Relation entre deux termes et stabilisation : le cas de want.
}

\author{
Olivier Polge
}

1 L'étude suivante vise à mettre en perspective l'opération de parcours d'une classe d'occurrences non validées dans la Théorie des Opérations Enonciatives en montrant qu'elle participe à certaines opérations de stabilisation, par exemple l'interrogation et non à d'autres, par exemple celle qu'implique le prédicat want, exprimant un manque soumis à une nécessité de remédiation qui a donné lieu à un fonctionnement principalementtransitif en anglais contemporain. Dans ce fonctionnement, le complément de rang zéro de ce verbe désigne un siège à propos duquel want prédique un manque et ce qui suit le prédicat désigne l'élément manquant, altérité non validée. Want exprime de nos jours le besoin ou le désir du siège du manque de valider l'objectif bénéfique qui est désigné par le terme qui suit le prédicat.

2 On commencera tout d'abord par définir le parcours d'une classe d'occurrences, énumérer ses formes possibles de stabilisation en ce qui concerne les inversions auxiliaire-sujet, les mots en WH et les énoncés mettant en place une relation intersubjective. Ensuite, après un aperçu de l'évolution diachronique des formes qui ont pu suivre le verbe want pour désigner l'élément manquant, on mettra en relation cette évolution diachronique avec les procédés de stabilisation évoqués pour les inversions auxiliaire-sujet, les mots en WH et les énoncés mettant en place une relation intersubjective.

\section{Définition du parcours et comparaison de ses formes de stabilisation avec celles d'une relation instable entre deux termes.}

3 A. Culioli (1991: 121) a donné une définition du parcours, reprise entre autres par J. Bouscaren et J. Chuquet (1987: 160) et E. Gilbert(1993: 90). Le parcours consiste à passer 
en revue l'ensemble de la classe d'occurrences associées à une notion envisagée, sans pouvoir ou vouloir valider une occurrence distinguée des autres occurrences de la classe. Par conséquent, il «ne peut se faire que sur du discontinu», selon J. Bouscaren et J. Chuquet (1987: 160), et implique donc l'existence d'une classe d'occurrences discrètes. Dans un article portant sur les inversions auxiliaire-sujet, R. Méry (1998) analyse les inversions auxiliaire-sujet et les mots en $\mathrm{WH}$ comme des marqueurs de parcours mais affirme (1998: 42) que l'opération de parcours appelle une "exigence de stabilisation ». Comme A. Culioli (1991: 121), il envisage quatre formes possibles de stabilisation par la séquence antéposée pour l'opération de parcours dont l'inversion auxiliaire-sujet est la trace. Les inversions auxiliaire-sujet et les mots en WH illustrent ces formes de stabilisation comme suit :

\begin{tabular}{|c|c|c|}
\hline Procédés de stabilisation & $\begin{array}{l}\text { Mots en WH (exemples de P. Larreya } \\
\text { et C. Rivière (2003)) }\end{array}$ & $\begin{array}{l}\text { Inversions } \\
\text { (exemples de R. } \\
\text { Méry (1998)) : }\end{array}$ \\
\hline $\begin{array}{l}\text { Relation inter-lexis par coréférence : } \\
\text { recours au contexte d'avant par } \\
\text { identification de l'élément } \\
\text { anaphorique par rapport à son } \\
\text { antécédent, à référent explicite }\end{array}$ & $\begin{array}{l}\text { relatives appositives: identification } \\
\text { du pronom relatif en WH à son } \\
\text { antécédent: } \\
\text { The Americans, who love baseball, are } \\
\text { not very keen on soccer. }\end{array}$ & $\begin{array}{l}\text { He would love to } \\
\text { go and so would } \\
\text { she. }\end{array}$ \\
\hline $\begin{array}{l}\text { Relation inter-lexis par corrélation: } \\
\text { stabilisation par identification } \\
\text { réciproque de deux variables, comme } \\
\text { dans: } \\
\text { The more he works, the more he earns. }\end{array}$ & $\begin{array}{l}\text { relatives déterminatives: } \\
\text { identification mutuelle entre le } \mathrm{TH} \\
\text { de l'antécédent et le WH ou TH du } \\
\text { relatif, image des antécédents } \\
\text { possibles: } \\
\text { The Americans who love baseball are not } \\
\text { very keen on soccer. }\end{array}$ & \\
\hline $\begin{array}{l}\text { Centrage : expression du haut degré } \\
\text { par renvoi au centre attracteur du } \\
\text { domaine notionnel }\end{array}$ & $\begin{array}{l}\text { Exclamatives: } \\
\text { How funny he is! }\end{array}$ & $\begin{array}{l}\text { Long did the } \\
\text { hours seem. }\end{array}$ \\
\hline $\begin{array}{l}\text { Recours à autrui pour fournir la bonne } \\
\text { valeur }\end{array}$ & $\begin{array}{l}\text { Questions en WH : } \\
\text { What made him so angry? }\end{array}$ & $\begin{array}{l}\text { Questions } \\
\text { fermées: } \\
\text { Did you sleep } \\
\text { well? }\end{array}$ \\
\hline $\begin{array}{l}\text { Passage à l'extérieur du domaine } \\
\text { notionnel }\end{array}$ & $\begin{array}{l}\text { Questions rhétoriques: } \\
\text { What else could I do? }\end{array}$ & $\begin{array}{l}\text { Séquence } \\
\text { antéposée } \\
\text { négative : } \\
\text { Never had he } \\
\text { heard such a } \\
\text { scream. }\end{array}$ \\
\hline
\end{tabular}

On s'aperçoit que ces différentes formes de stabilisation ont pour point commun le fait de poser l'existence d'une occurrence. 
D'après A. Culioli (1991: 170), le parcours précède la stabilisation d'une « relation entre deux termes instables». Il illustre ce type de relation par l'interrogation, qui peut être accompagnée par le marqueur donc en français. L'interrogation implique une opération de parcours avec les différentes formes possibles de stabilisation énumérées ci-dessus. Donc indique ce passage du parcours à l'occurrence validée. Dans le cas d'une interrogation monologuée ou rhétorique, donc renforce l'interrogation en provoquant un effet de surprise, d'impatience ou d'irritation. A. Culioli $(1991: 171)$ cite comme exemples :

Où donc est-il ? Où est-il donc?

Qui donc aurait pu prendre les clés?

Faudra-t-il donc que je me fâche?

6 A. Culioli (1991: 172) estime qu'une relation entre deux termes instables est aussi présente dans énoncés mettant en place une relation intersubjective :

7 Tout énoncé inter-subjectif marqué par un impératif (ou un subjonctif) représente une mise en relation entre deux positions, l'une qui renvoie à ce qui est le cas au moment où celui qui produit l'énoncé parle, l'autre qui renvoie à la représentation de létat de choses visé.

8 La relation intersubjective implique un parcours dans la mesure ou elle place l'agent potentiel dans une position où il doit choisir entre valider et ne pas valider le procès. Toutefois, la stabilisation de la relation intersubjective est envisagée par l'énonciateur qui imagine l'existence d'une occurrence, même si cette validation n'est que projetée dans l'avenir. En français, le marqueur donc dans un énoncé mettant en place une relation intersubjective permet à l'énonciateur d'insister sur le passage à la validation. A. Culioli (1991: 172) donne pour exemples :

Mettez-vous donc à l'aise !

Cesse donc de faire du bruit !

Dans le cadre de l'utilisation de want transitif, l'élément manquant dont la validation est recherchée est désigné par le terme qui suit le prédicat et dont la forme a subi une évolution au fil du temps.

\section{Evolution diachronique des formes qu'a pu prendre l'élément manquant.}

10 On remarque dans l'évolution diachronique de la construction transitive de want que l'élément qui suivait ce prédicat et qui désignait l'élément manquant a tout d'abord pris la forme d'un nom, puis a acquis la possibilité d'apparaître sous une forme en ING au XVIe siècle. Etymologiquement, cette forme en ING provient d'un nom de procès en UNG qui a été intégrée au paradigme du verbe à la fin du XIVème siècle tout en gardant des propriétés nominales.

11 Dans cette construction, le complément de rang zéro de want constitue l'argument but du prédicat en ING enchâssé. Cette construction a conservé jusqu'à nos jours un sens de besoin, comme dans l'exemple suivant :

(1) My very repentance needs repented of: and the tears I shed, want washing in the blood of Christ.

12 Ensuite, à partir de la fin du XVIIème siècle, est apparue la construction want to + base verbale : 
(2) 'I want topaint men and women with that something of the eternal which the halo used to symbolize,' Van Gogh had written, groping to define for his brother the human essence that was his aim in pictures such as this. (BNC) nécessité de remédiation qu'exprime want impose que cette forme renvoie à un processus télique, dont l'état résultant constitue une occurrence validée.

\subsection{Forme en ING renvoyant obligatoirement à un processus télique.}

Aucun verbe d'état ni de processus atélique n'a été rencontré dans cette construction où le verbe recteur want, suivi d'une forme en ING, a un complément de rang zéro renvoyant souvent mais pas forcément à de l'inanimé :

(4) 'Your hair wants cutting', said the Hatter.

'L. Carroll' Alice in Wonderland vii. (1866)

Dans cette construction, le prédicat enchâssé renvoie donc nécessairement à un processus télique. D'après la définition d'un processus par J.-P. Desclés in A. Culioli (2002 : 249), ce type de procès est évolutif. Il est composé de phases différentes qui permettent de passer progressivement de ce qui est le cas lors du constat de manque à l'état résultant du processus. On peut comparer cette définition à la définition topologique du parcours donnée par A. Culioli comme «trajet de point en point». Cependant, une opération de parcours s'effectue sur une classe d'occurrences indifférenciées tandis qu'un processus est constitué d'une classe d'instants distincts, l'instant d'après constituant un voisinage qualitativement différent de l'instant précédent. Pour les processus téliques, cette succession aboutit à l'état résultant, qui constitue une occurrence validée.

18 A partir de la fin du XVIIème siècle, un siècle après l'apparition de la forme en ING après want, on assiste à l'utilisation de plus en plus fréquente de la construction want to + prédicat, qui permet cette fois l'emploi de verbes considérés comme des verbes d'état ou renvoyant à des processus atéliques :

(5) I want to be a good vet who knows what she's doing. I haven't decided yet what I

want to specialise in. 
(6) I want to live with a husband who doesn't spend all his and our free time on the computer.

La présence de to correspond en fait à une opération de visée.

\subsection{Visée avec to $+\mathrm{V}$.}

La visée consiste à envisager l'existence future d'une occurrence de procès, d'après M.-L. Groussier et C. Rivière (1996: 165). La construction d'une occurrence, stabilisée sur les plans qualitatif et quantitatif, constitue donc un degré plus élevé de détermination du prédicat qui suit want qu'une forme en ING, qui ne stabilise l'occurrence de procès que sur le plan qualitatif, en renvoyant à la notion de participation d'un animé humain à ce procès.

21 Si l'on examine l'opposition entre to et ING dans la construction où want est suivi d'une proposition totalement instanciée, on s'aperçoit que la forme en ING est plus fréquente en présence d'une négation dans l'imbricante que sans la négation. Sur l'ensemble du BNC, deux cent énoncés contenant une forme en ING enchâssée après want apparaissant dans une assertion négative ont été recensées contre douze seulement dans une assertion positive. En effet, dans l'expression du rejet, la construction d'une occurrence n'est pas recherchée. A propos des énoncés sans négation, je rejoins G. Mélis (1994: 353) lorsqu'il affirme que ce type d'exemples n'implique pas la visée d'un état de fait mais une appréciation subjective arbitraire de la part du référent du complément de rang zéro de want, qui ne prend pas en compte d'autres opinions possibles, comme dans les exemples suivants :

(7) The Profitboss concentrates on the real ends of his business. He gets his satisfaction from contributing to high levels of profit, not from parading any status symbol the company cares to give him. He doesn't mind whether he's given the title of president, director or manager; these are mere status-labels All he minds about is making more and more profit. He knows that the pursuit of status can consume vast amounts of people's time and efforts with the negative result of demoralizing everyone around. The Profitboss is not interested in status. He parks with his team, eats with his team and uses the same toilets as his team. He takes care not to differentiate himself from his team in any way by status. He wants everyone pointing in the same direction, and that means a common culture and a common status. (BNC)

(8) I want you to talk to people. He looked slowly around the other faces in the room. I want pubs, clubs, clip-joints, restaurants and anything else you can think of, checked out. Talk to the staff. Two men have committed suicide within a one-mile radius of each other within a week. We've had a fucking chase through Soho and now a woman's been murdered. Somebody has seen something. Somebody knows something. I want that somebody found and I want them talking. (BNC)

(9) Thus, with a group of 32 children you'll have eight evenly balanced groups of four. What if you have a class of 32 and you want them working in groups of four, but today there are only 30 in school? Five groups of four and two of five, or six of four and two of three? Or seven groups of four and two doing something different? (BNC)

(10) "I'll go straight back to Eastbourne. I'll buy two identical coats and hats in some bright colour. Tomorrow I'll turn up here again at ten and tell the taxi to wait. I'll ask him to move along a bit, so he ain't blocking the entrance. I want him waiting by this corner. Then I come in with my suitcase, and Matron thinks I'm on my way to the airport. She's not to know I was supposed to catch yesterday's plane."

"Thank God you didn't." 
"In that suitcase, Nell, will be a coat and hat (same as mine) for you to wear. I get you dressed. I open them glass doors to the garden. You walk out slowly, with your head turned away from the house, to my taxi. You get in the taxi. I wait for you to do that; then I simply walk through the main hall, my empty suitcase in my hand. Then we drive hell for leather to the airport and catch the next plane to Nice."

"Buzz, darling Buzz, that's a brilliant escape plan." (BNC)

D'autre part, on remarque dans ces exemples que les pronoms qui instancient la place de premier argument des formes en ING sont dotés d'un référent peu déterminé. Il s'agit de pronoms indéfinis ou de pronoms personnels à la troisième personne, le plus souvent au pluriel. Même dans l'énoncé (10), où le pronom him est au singulier, le locuteur n'a pas encore rencontré le conducteur de taxi, dont l'identité importe peu et n'a aucune incidence sur le plan prévu. Le caractère peu déterminé du référent du pronom ne permet donc pas de mettre en place une véritable relation intersubjective, dans le cadre de laquelle l'énonciateur envisage la validation d'une occurrence et ce référent peu déterminé ne constitue donc pas l'origine spécifique nécessaire à l'apparition de to. La présence nécessaire d'une origine particulière dans le contexte pour l'apparition de to permet d'aborder la question de l'agentivité du référent du complément de rang zéro de want dans la construction want ... to.

\subsection{Le référent du complément de rang zéro a recours à autrui ou se projette dans l'avenir en tant qu'agent.}

Si le complément de rang zéro de want est doté d'un référent animé humain, celui-ci est susceptible de déclencher l'événement désigné par le verbe qui suit want. Or, J.-M. Merle (2003 : 160) considère que le complément de rang zéro de want n'est pas agent, mais siège du manque. Etant donnée cette absence d'agentivité, deux possibilités se présentent.

Soit le référent du complément de rang zéro de want met en place une relation intersubjective. La construction want someone to do something, où le référent de someone est nécessairement différent de celui du complément de rang zéro de want, apparait d'ailleurs vers 1750 . On citera comme exemple l'énoncé suivant :

(11) This week a gauche teenager came into the shop. He bluntly said that he wanted to see the boss--a lack of manners, no, a flimsy grasp of the language, yes. After I told him that the bookseller was not around, without any expressions he murmured, 'I want him to give me a job', and pulled out his CV only printed on half the size of an A4. Nationality: Poland... Utilities: Smiling... I've heard/read a lot on the news about the impact of the EU expansion after 1 May, but this is the first time I experienced it as close as on our doorstep.

Cette relation intersubjective est mise en place entre le référent du complément de rang zéro de want, coïncidant ici avec le locuteur et l'agent potentiel, ici le libraire. L'agent potentiel est susceptible de livrer une occurrence du procès give me a job.

Soit, en l'absence de tout autre agent potentiel, le référent du complément de rang zéro de want est interprété par défaut comme le déclencheur possible de l'occurrence du procès envisagé. Son statut de déclencheur est en fait provoqué par la présence de to dans le contexte. Au niveau des opérations, la place vide de premier argument de la forme to + V est identifiée au complément de rang zéro de want, qui est explicite. Cette opération est donc un phénomène de coréférence. 


\subsection{Coréférence et détermination mutuelle entre la place vide de premier argument de la forme to $+\mathrm{V}$ et le complément de rang zéro de want.}

27 Le phénomène de coréférence qui a lieu entre la place vide d'argument qu'implique la forme to $+\mathrm{V}$ et le complément de rang zéro de want ne constitue pas pour autant un cas d'effacement par coréférence du premier argument de la forme en to $+\mathrm{V}$. La place de premier argument de la forme to $+\mathrm{V}$ est vide à l'origine et elle est identifiée au référent $\mathrm{du}$ complément de rang zéro de want, présent dans le contexte avant.

Bien que le référent du complément de rang zéro de want soit explicite, il faut tout de même prendre en compte le fait que le complément de rang zéro de want et le terme qui le suit se déterminent mutuellement. On a vu que la construction d'une occurrence de procès dont to est la trace augmente le degré de détermination du prédicat qui suit want. Or, au niveau diachronique, l'apparition de to après want coïncide avec le développement de l'expression du désir par want transitif et l'expression du désir implique nécessairement un référent animé humain pour le complément de rang zéro de ce verbe. La présence d'un complément de rang zéro renvoyant à de l'animé humain, très déterminé, a donc pu provoquer la présence de to dans ce qui suit want. L'augmentation du degré de détermination du complément de rang zéro de want a par conséquent eu des répercussions sur le degré de détermination du prédicat qui le suit.

Inversement, on peut considérer que le référent du complément de rang zéro de want est déterminé par le prédicat qui suit want dans la construction want to si celui-ci renvoie à un procès qui lui confère le statut d'agent, même s'il n'est que projeté dans l'avenir.

\section{Conclusion.}

30 Parmi les procédés de stabilisation envisagés au départ pour le parcours d'une classe d'occurrences, la construction transitive de want illustre en tout cas le recours au contexte. L'expression du rejet par want,accompagné de la négation, mériterait un développement à part entière. Quoi qu'il en soit, certains procédés de stabilisation valables pour le parcours d'une classe d'occurrences, qui implique des opérations quantitatives de discrétisation, s'appliquent aussi à d'autres formes d'instabilité, qui sont dépourvues de ces opérations quantitatives de discrétisation.

31 A l'issue de cette étude, on rappellera le principe selon lequel tous les procédés de stabilisation décrits reviennent à poser l'existence d'une occurrence discrète, expression du rejet mise à part. En d'autres termes, même si une stabilisation peut ne pas donner lieu à un parcours d'occurrences discrètes, celle-ci est tout de même envisagée sous la forme d'une occurrence discrète, même si cette occurrence n'est que visée. C'est le cas dans l'énoncé suivant :

(13) There may also be a need for changes to criminal damage laws to cover viruses and logic bombs which can corrupt and destroy computer data. At the moment, under English law, data is not property, and damage or theft has to relate to the medium on which it is stored. But the main area of controversy is whether simply obtaining unauthorised access to a computer should be a crime. Emma Nicholson, Conservative MP for Devon West and Torridge, has lobbied that it should be, and for prison sentences of up to 10 years for culprits. The Confederation of British 
Industry also wants hacking outlawed, but the Data Protection Registrar is adamant that hacking per se should not be a crime. (BNC)

La forme de participe passé correspond à la validation d'une occurrence de procès même si want implique qu'elle est manquante et qu'elle n'est par conséquent que visée. Dans le cadre d'une représentation topologique, la stabilisation peut donc être considérée comme un mouvement orienté vers une occurrence validée.

\section{BIBLIOGRAPHIE}

BOUSCAREN, J., CHUQUET, J. et DANON-BOILEAU, L. (1987), Grammaire et textes anglais - Guide pour l'analyse linguistique, Ophrys, Paris.

CULIOLI, A. (1991), Pour une Linguistique de l'énonciation, Opérations et représentations, Tome 1, HDL, Ophrys, Paris.

CULIOLI, A. (2002), Variations sur la linguistique. Entretiens avec Frédéric Fau. Klincksieck, Paris.

GILBERT, E.(1993), « La théorie des Opérations Enonciatives d'Antoine Culioli », in Les Théories de la grammaire anglaise en France, COTTE, P. éd,Hachette Supérieur, Paris, pp. 63-96.

GROUSSIER, M.-L., et RIVIERE, C. (1996), Les mots de la linguistique, Lexique de linguistique énonciative ,Ophrys, Paris.

MELIS, G. (1994), Etude énonciative de l'opposition Base Verbale, Base Verbale + ING en anglais contemporain : facteurs contextuels et situationnels, Thèse de Doctorat, Université Paris VII.

MERLE, J.-M. (2003), « Want », in Linguistique comparée et traduction, Tome 6, Ophrys, Gap, pp. 151-181.

MERY, R. (1998), « Inversion sujet / auxiliaire avec antéposition d'un élément ni interrogatif ni négatif », in Anglophonia 10, Presses Universitaires du Mirail, Toulouse, pp. 33-71.

PEISER, C. (2004), What you Will is What you Want, Hausarbeit im Rahmen des ersten Staatsexamens für das Lehramt an Gymnasien, A semasiological study of the development of willan/will and want, Gutachter: Akad. Dir. Dr. Detlef Stark, Université de Hannovre.

\section{RÉSUMÉS}

L'opération de parcours d'occurrences non validées, qui sous-tend l'interrogation, par exemple, est stabilisée par divers procédés se ramenant pour la plupart à la validation d'une occurrence. Le verbe "want" à fonctionnement transitif est aussi suivi d'un terme renvoyant à une occurrence dont la validation est envisagée. Cette visée n'implique pas pour autant d'opération de parcours. Ce type de comparaison permet de considérer l'opération de parcours comme une simple étape vers la validation d'une occurrence. La présentation suivante vise donc à montrer que la stabilisation revient le plus souvent à poser ou viser l'existence d'une occurrence, qu'il y ait parcours ou non. 
The scanning operation of unvalidated occurrences, underlying interrogation for instance, is stabilised by various processes most of which boil down to the validation of an occurrence. The verb "want" with a transitive functioning is also followed by a term referring to an occurrence whose validation is envisaged. Still, this prospective validation does not involve any scanning operation. This kind of comparison makes it possible to regard the scanning operation as a mere stage towards the validation of an occurrence. The following presentation therefore aims at showing that stabilisation most often boils down to positing or contemplating the existence of an occurrence, whether scanning is involved or not.

\section{INDEX}

Mots-clés : procédés de stabilisation, validation d'occurrence, visée, relation intersubjective, siège du manque, déclencheur

Keywords : stabilising processes, validated occurrence, prospective validation, intersubjective relationship, experiencer, agent

\section{AUTEUR}

OLIVIER POLGE

UFR Charles V, Paris 7 